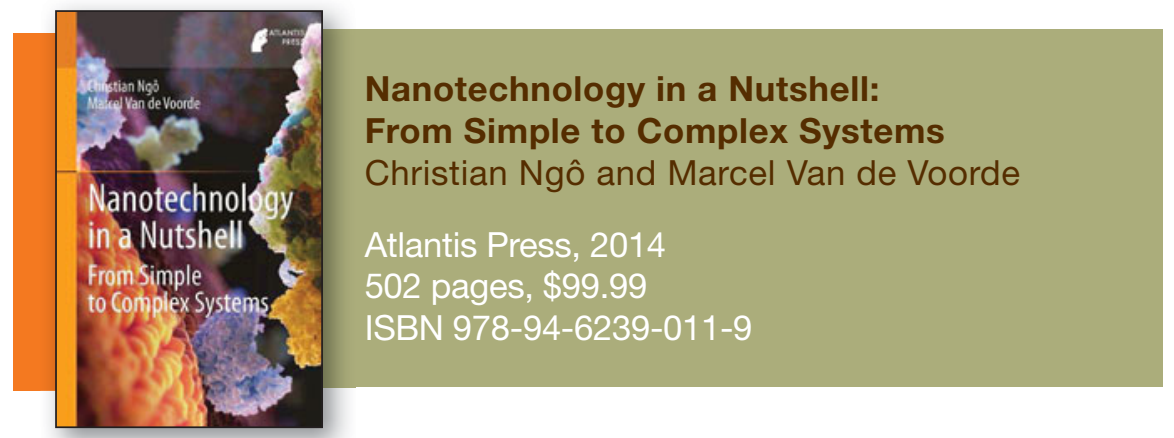

$\mathrm{T}$ his book offers an exciting insight into the fascinating nanoworld, from simple to complex systems. In this context, the authors have proven to be good interpreters of recent nanotechnology concepts useful to specialists acting in a wide pallet of interdisciplinary fields, as well as to the public at large. This type of information is vital for progress in the new technological age, as it presumes to produce a huge change in human thinking and mentality at individual and social levels, resulting in new life standards and even finding revolutionary solutions for saving life on our planet.
Starting from the classification of different generations of products coming from nanotechnology, the book succeeds in providing an overview of areas where nanotechnology is able to make substantial contributions, ranging from health care, environment cleaning, civil engineering, and security, to problems related to energy and sustainable communities. The volume is nicely illustrated by ingenuously designed schemes, and makes suggestive connections based on cause-and-effect relationships.

Moreover, the main merit and key points of this book stand in its accessibility to everyone interested in learning more about the potential miracles of nanotechnology; there are no intimidating formulas or mathematical proofs, but merely gentle explanations supported by attractive and relevant illustrations. This is probably why the reference list is rather short and limited to books and monographs in various applied fields, and contains no articles published in international scientific journals. However, it is still a large volume of information, and to cover everything effectively is not a simple task, yet the authors succeed in doing so in an elegant manner.

At this general level of information, this work is a rich source of inspiration for many specialists, directing them to cutting-edge ideas and connecting them to unexpected innovations and applications in complementary research and technology.

Reviewer: Aurelia Meghea is Professor Emeritus at the University Politehnica of Bucharest, Romania.
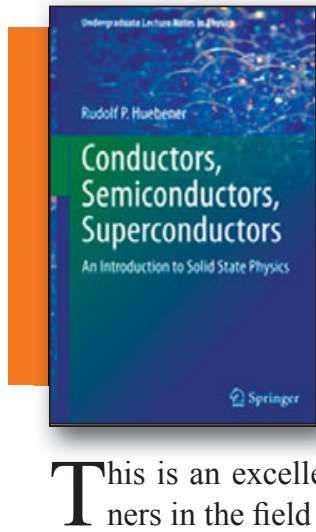

Conductors,

Semiconductors,

Superconductors

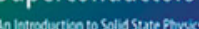

\section{Conductors, Semiconductors, Superconductors: An Introduction to Solid State Physics Rudolf P. Huebener}

Springer, 2014

215 pages, $\$ 59.99$

ISBN 978-3319091402 structure, diffraction theory, and the types of bonding between the atoms $/ \mathrm{mol}-$ ecules and the crystal properties originating from lattice vibrations. In the fourth chapter, a quantum mechanical approach is employed to describe the difference between insulators, semiconductors, and conductors. The roles of the Fermi surface and momentum space in electronic properties of materials are presented in the fifth chapter. Intrinsic and extrinsic semiconductors and their applications are well discussed with the band model in the sixth chapter. Electronic motion in a magnetic field and related experiments are described in the seventh chapter. The eighth and ninth chapters contain the fundamentals of superconductivity and the invention of high-temperature superconductivity with beautiful images and explanations. The tenth chapter explains different types of magnetism and magnetic materials along with their applications. The most advanced achievements and recent topics in nanotechnology, such as topological insulators, are discussed in the last chapter to give a brief introduction to contemporary research fields.

Fundamentals of solid-state physics along with the most recent discoveries and research topics are very well written in this book. A few notations used in the equations have a slightly different approach compared to the usual way of writing them. Learning science along with the history about the scientists and their groundbreaking discoveries will make a positive impact on bachelor students' perceptions toward the research field.

Reviewer: K. Kamala Bharathi of the National Institute of Standards and Technology/University of Maryland, Gaithersburg, Md., USA. cal tools. The second and third chapters deal with the fundamentals of crystal 